$\mathbb{T}$ periodica polytechnica

Civil Engineering

$54 / 1(2010) 6165$

doi: 10.3311/pp.ci.2010-1.07

web: http://www.pp.bme.hu/ci

(c) Periodica Polytechnica 2010

RESEARCH ARTICLE

\section{Road network vulnerability-evaluation of measures in ranking damages and developments}

\author{
Gábor Schuchmann
}

Received 2009-09-21, accepted 2009-10-16

\begin{abstract}
In this article a practical example on studying vulnerability using the method of the critical scenario and multi-criteria analysis is presented. Giving a simple method to handle reliability related to a certain path, section, link, junction or a whole trip for travelers and operators of urban road networks as users of traffic information systems it would be a huge step forward. The best would be to have one single parameter coming from an engineering calculation or a model, which would mean the same for the user and the engineer, and both of them would be able to use it as it was. Introducing a clear definition of reliability and vulnerability of road networks used by a wide range of applications is of capital importance. This article makes some steps towards achieving this goal.
\end{abstract}

\section{Keywords}

Transportation networks - vulnerability · critical scenario . simulation model $\cdot$ weighted multi-criteria decision

\section{Gábor Schuchmann}

Department of Highway and Railway Engineering, BME, H-1111 Budapest, Múegyetem rkp. 3., Hungary

e-mail: schuchmann@uvt.bme.hu

\section{Introduction}

The vulnerability of a road network became the subject of attention since 1995, The Great Hanshin Earthquake in Kobe, Japan. There are many serious threats that can cause urban or rural road networks to fail or to become severely impeded (congestion, natural hazards, structural breakdowns, traffic accidents, traffic management failures, to mention but a few).

Road network is one of the most important and most complicated part of a modern society. Consequently, the reliability of a road network is a decisive factor in terms of market outreach and competition as well as continuity, to ensure a $24 / 7$ operation for the society we live in. Any threat bringing down reliability of the road network means a vulnerable spot (weakness). By urban standards it can cause a minor degradation (i.e. car accident, resulting in queuing, delays and diversions), but the consequences can be more serious if it is a rural case (i.e. when an avalanche blocks the only access road for a long time, even days or weeks).

An average user of the transportation network wants to know the answer of the following question: Knowing the desired time of departure, is it possible to get from A to B by using a certain route and means of transport, and arrive at a desired time? Is there no route or means of transport at all that can take me from A to B at that time of departure or within arriving at the desired time (no user likes this „worst case” answer). A freight hauler thinks a network vulnerable that is easily disrupted, resulting in unpredictable stops and downtime. This is a more difficult problem than a congested network which is reliably slow, meaning the goods will arrive, costs are calculable and the arrival time at the destination is predictable (even though a haul on this kind of network will not be the most efficient one).

The unpredictably and quickly collapsing elements or network parts can cause unreliable route plans (even the dynamic ones) for the user while the traffic operation system have no chance for either a good prediction or an appropriate reaction (even when these are based on momentary information).

The vulnerability of transport networks does not yet have a commonly accepted definition and/or methodology, and the scientific base behind the discussion and the problem itself is missing. Most of the authors agree that the key to vulnerability re- 
search is the analysis and study of the impacts of the different threats to the network (and not the threats themselves). Accordingly, analyzing the future impacts of possible threats, the vulnerability analysis must focus on three important questions: Where is the network vulnerable? Vulnerable to what? How is it vulnerable?

In some cases there is no chance to find an answer for a question of these three without answering at least another, sometimes all three. On the other hand, when trying to draft the answer it is easy to realize that different words sometimes mean the same thing when trying to explain, and in worse cases the similar word is used for a different meaning.

The key to find the answers to the questions was to study all the possible known aspects of vulnerability, thus the link weakness index was analyzed first. This index shows ,how important each link is for the overall set of origin/destination pairs, by assessing how many o/d paths share the same link" [1]. By weighing the weakness index with travel demand, the link exposure index can be calculated for each link. For the second try [2] the increment in generalized travel costs weighted by the satisfied or unsatisfied demand when network links are closed was used as a measure of vulnerability. Importance reflects the significance of each link with regard to the network, and exposure reflects the increment in travel cost for a given location within the network. Together these measures provide substantial information like the location of the most vulnerable (exposed and or/important) links in the network [3]. In this article the critical scenario method is implemented and weighted multi-criteria decision approach is applied. These methods can be described as ,link closures or degradations are assessed by various categories of effect and the severity of the impact, thus allowing for the assessment of individual effects or impacts" [4].

\section{Concept}

The concept of this article is to study the correlation of some (i.e. traffic, environmental, network) parameters and the vulnerability metrics given by some authors brave enough to make a suggestion for the context before anybody else. The two questions of mine to find the answer for, while analyzing how useful each metric of vulnerability can be to evaluate some problems of network operation and development were (the answer for these questions was tried to find before with a different approach in [5]):

1 Is it possible to make an order for getting over the damages using the analyzed two metrics?

2 Is there an appropriate metric of vulnerability that can help in choosing between certain developments scenarios?

To find the answer to these questions above it was necessary to

- Build an appropriate network model;

- Find traffic parameters for each metric of vulnerability;

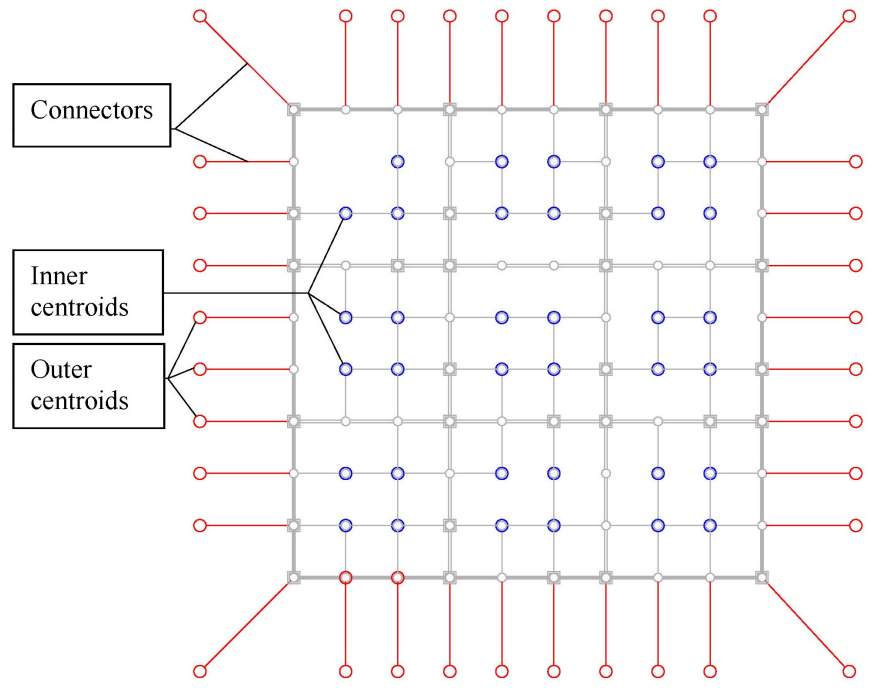

Fig. 1. Network model geometry

- Analyze the correlation between the traffic parameter and the metric of vulnerability;

- Find out how it is possible to use the context proven in decision making, route planning and network development.

The base and the most important tool of these studies was a database of a network simulation model [6-9]. The database generated by the simulation contains all the data needed to analyze the connection between any given parameter or set of the parameters (i.e. speed, follow-up distance, density, etc.) and the metrics given by the theory waiting for to be proved (i.e. weakness, exposure, importance, etc.). The base of the simulation itself is a continuous data flow of certain parameters like follow-up distance as a function of lane speed and density coming directly from special software processing traffic surveillance camera pictures [10].

\section{A model of developments and damages}

To analyze the vulnerability metrics a transportation network model must be built which provides all the data needed to find all the contexts wanted. This model is a grid of 10 rows and 10 columns, with a link length of 1000 meters (see Fig. 1). This model is supposed to be the main road network of a city with only multilevel and signalized intersections. There are 9 different scenarios of geometry (named V1...V9) where the type of the intersection, the number of lanes and the speeds are different in each. These scenarios supposed to represent 9 different levels and states of development in the life of a city.

All the scenarios were assigned using the same set of ,damages" causing smaller or bigger restrictions on certain elements in some traffic parameters (lane closure, sectional speed limits, turn penalties, etc.). There were three forms of damages (see Fig. 2):

1 Extended damages (R1a: main road total closure for example because of flood; R1b: downtown is closed for example 
because of security reasons);

2 Smaller, occasional damages (for example accident in an intersection);

3 Sets of different damages (road reconstructions).

In the case of R1a a main road is not available for traffic. In some cases the capacity of the damaged main road related to the network is big enough to collapse the whole traffic of the network. If only a hard reduction of the road section to one lane per direction is applied, extremely long travel times on the network are avoidable. In case R1b (downtown closure) accessibility of the closed area remains as it was because the damaged network must be assigned with the same matrix as it was before, thus surrounding main roads continue operating with reduced conditions (1 lane per direction, speed limits and hard turn penalties at intersections).

Damages R2 are modeled with changes in the regulation of the intersection with lane redistribution and different speed limits as increasing intersection crossing penalties. Considering R3 damages there is always at least one lane open for traffic along the road works without speed limitations.

\section{Methodology}

First the critical scenario approach was implemented to find the answer for the first question. (Is it possible to make an order for getting over the damages using any of the known metrics?) Of course the real question when managing a road network is which damage has to be dissolved first to help the network as much as possible. To find the answer the scenario (damage or set of damages) causing the biggest travel time increment on the network had to be found, and an order of the scenarios had to be given. These three forms of damages (R1, R2 and R3) generate three questions when trying to answer the first one:

1 Which of the two typical R1 damages causes bigger travel time increment on the network?

2 Which one of a given group of R2 damages causes the biggest travel time increment on a network?

3 Which combination of a special set of R3 damages causes the smallest travel time increment on the network?

Secondly the multi-criteria analysis was applied in accordance with Husdal's suggestion [3]. In this case link closures or degradations are assessed by various categories of effect and the severity of the impact, thus allowing individual effects or impacts for the assessment. Using the flexibility of this method as many parameters as possible were fixed, and the scenarios meaning special sets of developments were compared. To be able to compare different developments the increment of total travel time on the network was used as a measure of vulnerability. Accordingly showing the travel time decrease between two scenarios as well as ranking them by cost-efficiency was possible from the same database.

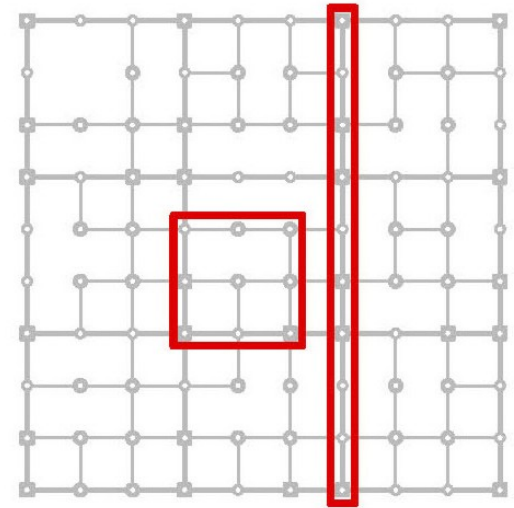

Form R1

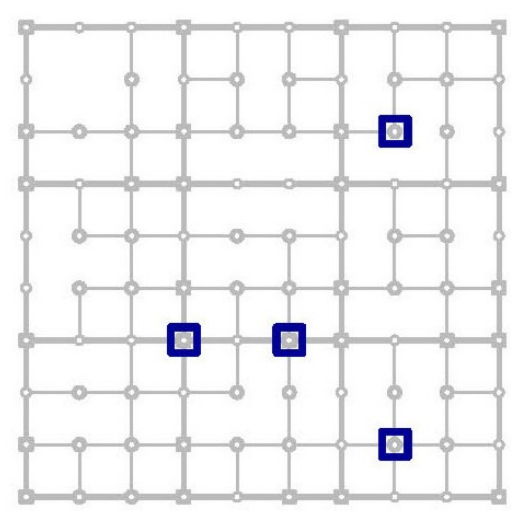

Form R2

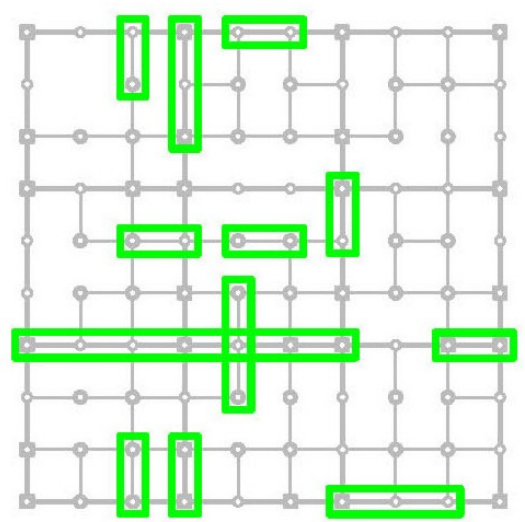

Form R3

Fig. 2. Forms of damages 
Tab. 1. Total travel time changes caused by R1 damages [million seconds]

\begin{tabular}{cccc}
\hline Scenario & $\begin{array}{c}\text { Without } \\
\text { damages }\end{array}$ & With R1a & With R1b \\
\hline V1 & 44.77 & 59.99 & 65.81 \\
\hline V2 & 38.26 & 50.51 & 54.72 \\
\hline V3 & 36.10 & 46.93 & 49.09 \\
\hline V4 & 38.93 & 47.89 & 49.05 \\
\hline V5 & 36.73 & 44.81 & 45.54 \\
\hline V6 & 37.62 & 44.02 & 45.14 \\
\hline V7 & 35.16 & 41.14 & 42.19 \\
\hline V8 & 33.49 & 39.18 & 40.18 \\
\hline V9 & 34.15 & 39.96 & 40.98 \\
\hline
\end{tabular}

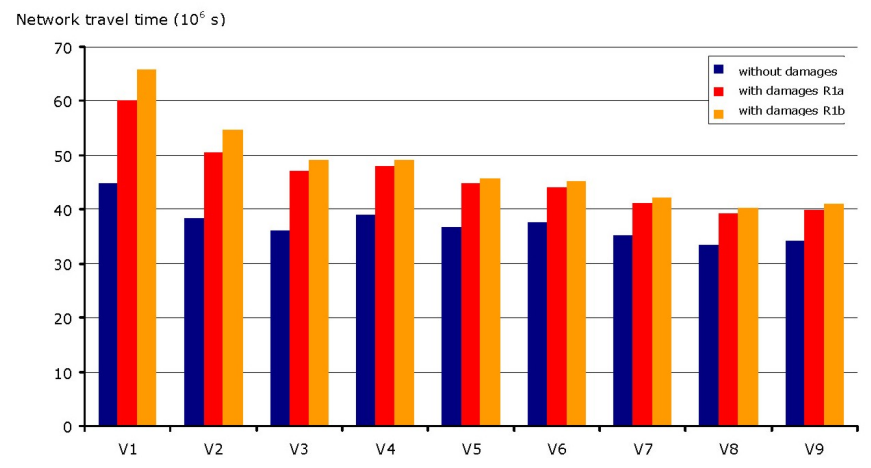

Fig. 3. Total travel time changes caused by R1 damages (as in Table 1 ,

\section{Results}

After defining all the developments (scenarios) and damages, the network model was assigned with several matrices to have as many states of all the elements as possible. After approximately 8750 assignments on the 9 scenarios of the network with all the possible sets of damages, the connection between the physical meaning of the vulnerability metrics and the traffic parameters given by the model was found.

\subsection{Damages}

Analyzing the differences in travel times on Rl type damaged networks it is clear that the relative increment in travel times is smaller when causing it to a more developed scenario of the network (see Table 1 and Fig. 3). The last 3 cases have practically the same total travel time, because of the same total capacity (only intersectional developments were applied).

Looking at the differences in travel times on $R 2$ type (intersectional) damaged networks it became obvious that for smaller intersections this method will not give any valuable results: one of the four tries gave a difference of $0.02 \%$ (means nothing), another around $0.5 \%$ (inside the $0.6 \%$ statistical gap of the simulation-based traffic forecasting). The other two damages (purposely in two intersections with heavy traffic) caused some $0.5-1.0 \%$ travel time increment on the first 3 scenarios, but on

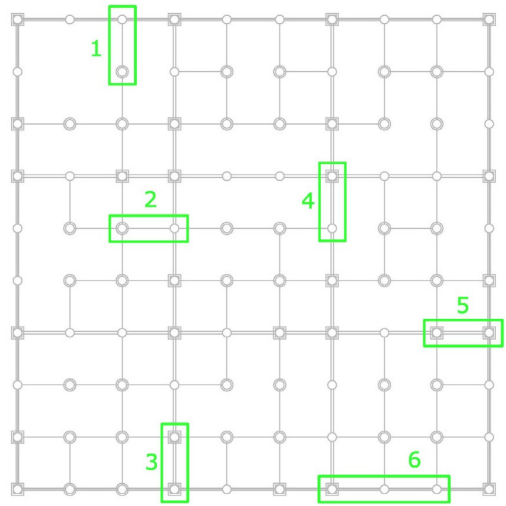

Fig. 4. R3 damages on the network

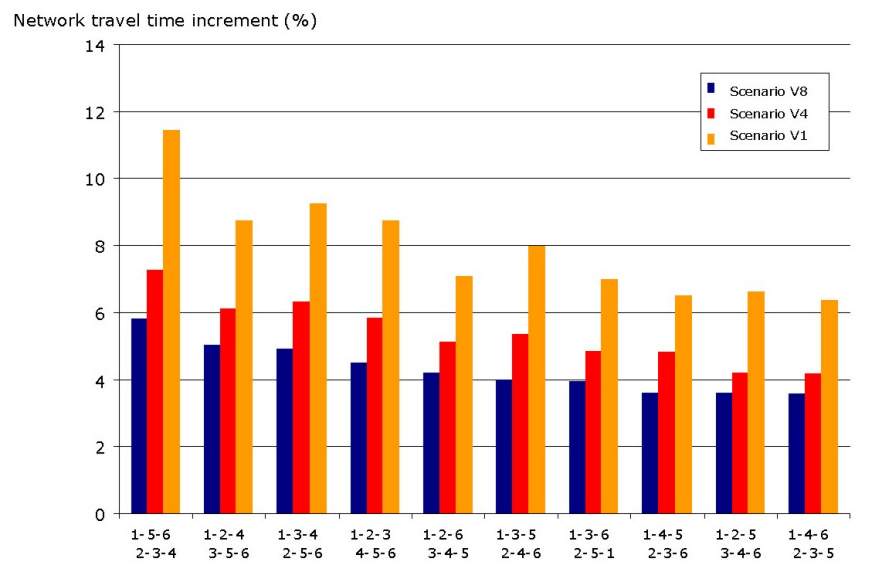

Fig. 5. Relative network travel time increment by all $3+3$ combinations of R3 damages on scenarios V1, V4 and V8

the other scenarios less than $0.6 \%$.

Testing the effects of $R 3$ type damages a package of 6 damages was created on the network (see Fig. 4), and created all the possible $3+3$ combinations of them (there are 10 of these) to analyze them on three scenarios (V1, V4 and V8). The results are in Table 2 and Fig. 5

\subsection{Developments}

Comparing the scenarios with the multi-criteria analysis method all of them showed a certain amount of travel time decrease related to the previous one (Fig. 6). To have a second criteria an estimated cost was calculated for each development to compare the cost-effectiveness (normalized costs) of all the scenarios (Fig. 7).

\section{Conclusion}

The results on network damages showed that it is possible to make an order for getting over (or causing) certain damages using generalizing the critical scenario method to get the travel time increment of the network. This tool is available for the network operators to minimize the time loss caused by necessary road work or other restrictions on the network.

On the other hand the possibility of ranking developments based on their normalized costs with the multi-criteria analysis 
Tab. 2. Relative network travel time increment by all $3+3$ combinations of R3 damages on scenarios V1, V4 and V8 [\%]

\begin{tabular}{lccc}
\hline Combination & Scenario V8 & Scenario V4 & Scenario V1 \\
\hline $1-5-6-2-3-4$ & 5.80 & 7.26 & 11.46 \\
\hline $1-2-4-3-5-6$ & 5.05 & 6.14 & 8.74 \\
\hline $1-3-4-2-5-6$ & 4.90 & 6.33 & 9.25 \\
\hline $1-2-3-4-5-6$ & 4.51 & 5.82 & 8.73 \\
\hline $1-2-6-3-4-5$ & 4.21 & 5.14 & 7.08 \\
\hline $1-3-5-2-4-6$ & 4.00 & 5.37 & 7.98 \\
\hline $1-3-6-2-5-1$ & 3.95 & 4.85 & 6.99 \\
\hline $1-4-5-2-3-6$ & 3.61 & 4.81 & 6.52 \\
\hline $1-2-5-3-4-6$ & 3.59 & 4.21 & 6.65 \\
\hline $1-4-6-2-3-5$ & 3.57 & 4.19 & 6.37 \\
\hline
\end{tabular}

Network travel time decrease $(\%)$

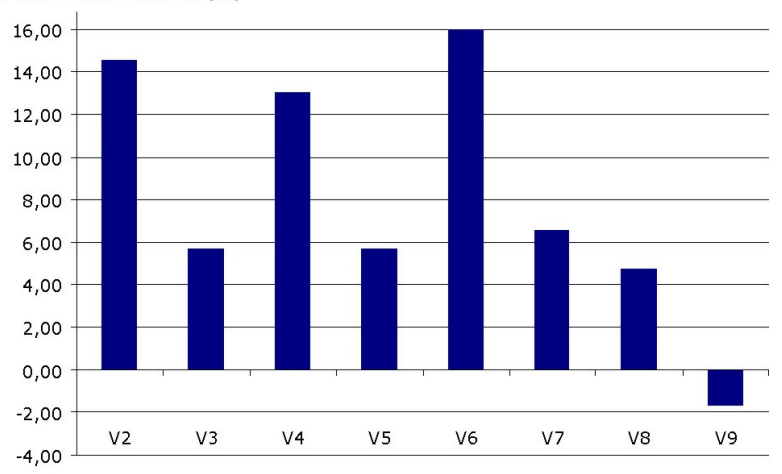

Fig. 6. Network travel time decrease (related to the less developed ones)

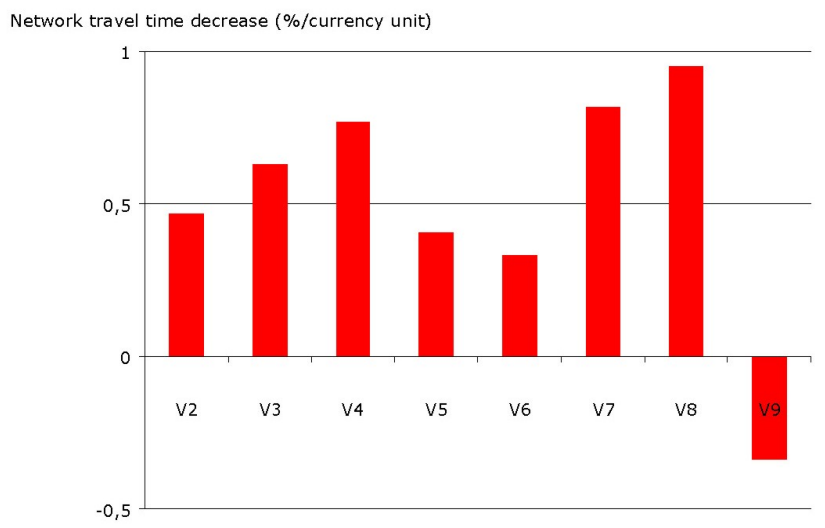

Fig. 7. Network travel time decrease can be bought for the same amount of money method using the travel time increment was shown. Accordingly when making an order of developments decision makers have an easy-to-use and network-independent tool to give the best possible network to the society that will use it.

\section{References}

1 Di Mangi M, Luongo A, Transportation Network Vulnerability Indicator for Risk Evaluation and Exposure Reduction, Proceedings of the European Transport Conference (2005).

2 Schuchmann G, Aspects of transportation network vulnerabilityapplication limits of the general transportation costs method, Proceedings of ÉPKO 2009 ( 2009), 420-427.

3 Jenelius E, Petersen T, Mattsson L, Transportation Research Part A: Policy and Practice, 7, vol. 40, Elsevier, 2006.

4 Husdal $\mathbf{J}$, The vulnerability of road networks in a cost-benefit perspective, Proceedings of the TRB Annual Meeting (Washington DC, USA, 2005).

5 Schuchmann G, Assessment of bus priority systems' efficiency, Periodica Polytechnica 48 (2005), no. 1-2, 133-140.

6 Siki Z, Simulation of road networks, BUTE, 1995.

7 Bécsi T, Péter T, Development and Evaluation of a Fuzzy-based Microscopic Vehicle-following Model, Periodica Polytechnica Ser. Transp.Eng. 36 (2008), no. 1-2, 15-20, DOI 10.3311/pp.tr.2008-1-2.03.

8 Schreckenberg M, Schadschneider A, Nagel K, Ito N, Discrete stochastic models for traffic flow, Physical Review E 51, posted on 1995, 2939-2949, DOI 10.1103/PhysRevE.51.2939, (to appear in print).

9 Dijkstra E, A note on two problems in connexion with graphs, Numerische Mathematik 1 (1959), no. 1, DOI 10.1007/BF01386390.

10 Czuczor Sz, DugóFigyu: Video based, robust road traffic evaluating system, Proceedings of KÉPAF (Debrecen, Hungary, 2007), pp. 200-206. 